\title{
Reply to the Letter to the Editor: "Comparison of the Safety and Efficacy of Yttrium-90 Radioembolization for Nonalcoholic Fatty Liver Disease-Associated and Hepatitis B Virus-Associated Hepatocellular Carcinoma"
}

\author{
Clemens Schotten $^{a} \quad$ Heiner Wedemeyer ${ }^{a} \quad$ Jan Best $^{\mathrm{a}}{ }^{\mathrm{b}}$ \\ a Department of Gastroenterology and Hepatology, Essen University Hospital, Essen, \\ Germany; ${ }^{b}$ Department of Gastroenterology, Hepatology and Infectious Diseases, Otto von \\ Guericke University Magdeburg, Magdeburg, Germany
}

Dear Editor, dear colleagues,

We would like to express our gratitude for the valuable comments that the authors gave in response to our manuscript entitled: "NAFLD-Associated Comorbidities in Advanced Stage HCC Do Not Alter the Safety and Efficacy of Yttrium-90 Radioembolization" [1]. We fully agree with the colleagues that propensity score analysis is potentially capable to reduce a selection bias and thus decreases the likelihood of confounding when analyzing nonrandomized data [2-6].

Recent publications confirm that patients with nonalcoholic fatty liver disease (NAFLD)-associated hepatocellular carcinoma (HCC) exhibit poorer survival when subjected to standard-of-care therapy. This effect is most likely attributable to the extent of metabolic comorbidities and late-stage HCC diagnosis [7-10].

To elucidate the potential negative effects of NAFLD-related metabolic and cardiovascular comorbidities on selective internal radiotherapy, a matching control cohort was required. To address this, patients with an identical tumor stage in the absence of equivalent comorbidities had to be identified. HCC patients with chronic hepatitis B virus (HBV) infection were identified who exactly fulfilled those requirements mentioned.

In the authors' opinion, utilization of propensity score analysis would add limited benefit to our study, for the following two reasons:

- Differences in disease characteristics between NAFLD and HBV infection were chosen intentionally to have a cohort with a high level of metabolic comorbidities (NAFLD) to compare with another cohort revealing significantly fewer comorbidities (HBV)

- Propensity score analysis can only include confounders that are observed; hence, the propensity score model could not control for confounders that were not observed

Propensity score analysis is a well-established method for examining data from nonrandomized pharmacological studies. However, for our study, this method adds limited benefit to a multivariate regression model.

Patients with NAFLD and HBV infection exhibited distinct differences in baseline characteristics, while BCLC stage incorporating tumor stage, liver function, and performance status revealed no significant 
difference. The ALBI score, representative of overall liver function, also did not differ significantly between the NALFD and the HBV cohort.

Propensity score matching is a well-validated method; however, for our purposes, it would have decreased the statistical power without providing additional insights into NAFLD. The primary aim of our study was to investigate whether NAFLD-associated comorbidities alter the safety and efficacy in selective internal radiotherapy. Using a matching method to overcome the chosen differences between NAFLD and HBV infection was not within the scope of this study.

\section{Disclosure Statement}

J. Best received a speaker's fee from BTG (speakers bureau, consulting). H. Wedemeyer received personal fees from AbbVie, Abbott, Janssen, Eiger, MYR GmbH, Falk, Norgine, and MSD, and personal fees and financial support from Abbott, BMS (Consulting, Research Support, speakers bureau), Gilead, and Roche Diagnostics.

\section{Funding Sources}

No funding was received.

\section{Author Contributions}

Conception: C. Schotten, H. Wedemeyer, and J. Best; manuscript preparation: C. Schotten and J. Best.

\section{References}

1 Schotten C, Bechmann LP, Manka P, Theysohn J, Dechêne A, El Fouly A, et al. NAFLD-associated comorbidities in advanced stage HCC do not alter the safety and efficacy of yttrium-90 radioembolization. Liver Cancer. 2019 Nov;8(6):491-504.

2 Liang L, Sun LY, Diao YK, Yang T. Comparison of safety and efficacy of yttrium-90 radioembolization for nonalcoholic fatty liver disease-associated and hepatitis B virus-associated hepatocellular carcinoma. Liver Cancer. DOI: $10.1159 / 000506353$.

3 Kuss 0, Blettner M, Börgermann J. Propensity score: an alternative method of analyzing treatment effects. Dtsch Arztebl Int. 2016 Sep;113(35-36):597-603.

4 Ali MS, Prieto-Alhambra D, Lopes LC, Ramos D, Bispo N, Ichihara MY, et al. Propensity score methods in health technology assessment: principles, extended applications, and recent advances. Front Pharmacol. 2019 Sep; 10:973.

5 King G, Nielsen R. Why propensity scores should not be used for matching. Polit Anal. 2019;27(4):435-54.

6 Winkelmayer WC, Kurth T. Propensity scores: help or hype? Nephrol Dial Transplant. 2004 Jul;19(7):1671-3.

7 Weinmann A, Alt Y, Koch S, Nelles C, Düber C, Lang H, et al. Treatment and survival of non-alcoholic steatohepatitis associated hepatocellular carcinoma. BMC Cancer. 2015 Apr;15(1):210.

8 Jun TW, Yeh ML, Yang JD, Chen VL, Nguyen P, Giama NH, et al. More advanced disease and worse survival in cryptogenic compared to viral hepatocellular carcinoma. Liver Int. 2018 May;38(5):895-902.

9 Guo H, Wu T, Lu Q, Dong J, Ren YF, Nan KJ, et al. Hepatocellular carcinoma in elderly: clinical characteristics, treatments and outcomes compared with younger adults. PLoS One. 2017 Sep;12(9):e0184160.

10 Williet N, Clavel L, Bourmaud A, Verot C, Bouarioua N, Roblin X, et al. Tolerance and outcomes of sorafenib in elderly patients treated for advanced hepatocellular carcinoma. Dig Liver Dis. 2017 Sep;49(9):1043-9. 